\title{
Computer-assisted virtual planning and surgical template fabrication for frontoorbital advancement
}

\author{
*Jehuda Soleman, MD, ${ }^{1}$ Florian Thieringer, MD, DDS, MHBA, ${ }^{2}$ Joerg Beinemann, MD, ${ }^{2}$ \\ Christoph Kunz, MD, DDS, ${ }^{2}$ and Raphael Guzman, MD'

\begin{abstract}
1Department of Neurosurgery, Division of Pediatric Neurosurgery, and 'Department of Cranio-Maxillofacial Surgery, University Hospital of Basel, Switzerland
\end{abstract}

\begin{abstract}
OBJECT The authors describe a novel technique using computer-assisted design (CAD) and computed-assisted manufacturing (CAM) for the fabrication of individualized 3D printed surgical templates for frontoorbital advancement surgery.

METHODS Two patients underwent frontoorbital advancement surgery for unilateral coronal synostosis. Virtual surgical planning (SurgiCase-CMF, version 5.0, Materialise) was done by virtual mirroring techniques and superposition of an age-matched normative 3D pediatric skull model. Based on these measurements, surgical templates were fabricated using a 3D printer. Bifrontal craniotomy and the osteotomies for the orbital bandeau were performed based on the sterilized $3 \mathrm{D}$ templates. The remodeling was then done placing the bone plates within the negative 3D templates and fixing them using absorbable poly-DL-lactic acid plates and screws.
\end{abstract}

RESULTS Both patients exhibited a satisfying head shape postoperatively and at follow-up. No surgery-related complications occurred. The cutting and positioning of the 3D surgical templates proved to be very accurate and easy to use as well as reproducible and efficient.

CONCLUSIONS Computer-assisted virtual planning and 3D template fabrication for frontoorbital advancement surgery leads to reconstructions based on standardized measurements, precludes subjective remodeling, and seems to be overall safe and feasible. A larger series of patients with long-term follow-up is needed for further evaluation of this novel technique.

http://thejns.org/doi/abs/10.3171/2015.3.FOCUS14852

KEY WORDS craniosynostosis; frontoorbital advancement; virtual surgical planning; 3D printed templates

$\mathrm{C}$ RANIOFACIAL remodeling surgery for craniosynostosis is complex and presents a multidisciplinary challenge. ${ }^{21}$ The indication for surgery is mainly to correct the cranial deformity, but the potential presence of elevated intracranial pressure and psychological disadvantage caused by untreated craniosynostosis are crucial as well. ${ }^{7,820}$ The challenge of frontoorbital advancement surgery is to meticulously reshape the orbital bandeau and frontal bone flap while accounting for individual variations. ${ }^{8,19}$ The reconstruction of the orbital bandeau, the amount of advancement, and the forehead reconstruction rely mostly on an individual surgeon's experience. Despite the application of best practice, there is a relapse rate of up to $65 \%$ described in the literature..$^{11,23,24,28} \mathrm{In}$ an attempt to improve the reproducibility of the frontoorbital recon- struction, standardized methods have been described and evaluated..$^{6,8,19}$ In addition, operation time and blood loss could be minimized through a standardized procedure. ${ }^{6,26}$

Modern approaches have tried to apply image-based 3D modeling using computer-assisted design (CAD) and computed-assisted manufacturing (CAM) of implants to create surgical templates. Normative 3D pediatric skull models have been analyzed and can be used for preoperative planning. ${ }^{15,22}$ The remodeling procedure is not defined beyond some general rules, and an objective outcome assessment is almost impossible. ${ }^{8}$ Lately, systems relying less on subjective assessments and more on standardized measurements using age-matched templates and CAD/ CAM techniques for preoperative planning in surgical correction of craniosynostosis have been described. ${ }^{1,10,16,25}$

ABBREVIATIONS CAD = computer-assisted design; $C A M=$ computer-assisted manufacturing; PDLLA = poly-DL-lactic acid.

SUBMITTED January 1, 2015. ACCEPTED March 4, 2015.

INCLUDE WHEN CITING DOI: 10.3171/2015.3.FOCUS14852.

DISCLOSURE The authors report no conflict of interest concerning the materials or methods used in this study or the findings specified in this paper.

* Drs. Soleman and Thieringer contributed equally to this work. Drs. Kunz and Guzman share senior authorship. 
Computer-based remodeling analysis of each individual child and creation of individualized, 3D printed surgical guides can help standardize these complex reconstructive procedures and potentially lead to a better surgical outcome. In this study we describe a novel technique in which we use computer-assisted procedures for fabrication of individualized 3D printed surgical templates for frontoorbital advancement surgery.

\section{Methods}

\section{D Modeling and Virtual Correction Process}

Our patients underwent thin-cut CT scanning with 3D reconstructions of the head to evaluate the craniosynostosis as well as facial and skull base deformities. An optimized protocol for infants was applied with radiation dose reduction (Table 1). Special attention was directed to include the complete soft-tissue volume into the scan of the skull (anterior, tip of nose; posterior, occiput; cranial, vertex; and caudal, occipital condyles and maxillary alveolar process). This was important for subsequent correct virtual 3D-based intervention planning and detection of all deformation simulations, including symmetry investigations. The infant's head was stabilized during CT scanning, paying special attention to avoid pressure deformation of the facial skin. The DICOM data set was transferred to a computer system with extended graphic card (GeForce GTX 640 graphic chip, NVIDIA), imported, and $3 \mathrm{D}$ reconstructed using the software SurgiCase-CMF (version 5.0, Materialise) (Fig. 1). After visualization of the skull morphology, the 3D virtual planning was performed. The procedure was started by outlining the classic cranial osteotomies for a frontoorbital advancement (bifrontal craniotomy and orbital bandeau; Fig. 2). First, we used mirroring techniques of the patient's unaffected side (Fig. 3A and B). For further correction, near-similar reference skulls of patients with the same age, sex, and ethnicity, free of alterations or deformations in relevant regions, and without any scanned artifacts were used (Fig. 3C). The virtual orbital bandeau and the frontal bone were

TABLE 1. Applied CT parameters

\begin{tabular}{ll}
\hline \multicolumn{1}{c}{ Parameter } & \multicolumn{1}{c}{ Description } \\
\hline No. of images & $\begin{array}{l}206-234 \\
\text { Protocol }\end{array}$ \\
$\begin{array}{ll}\text { CT using the skull/CCT-coil in infants } \\
(0-5 \mathrm{~kg}) ; \text { noncontrast scan }\end{array}$ \\
\hline Pixel size & $0.3691-0.3965 \mathrm{~mm}$ \\
\hline Resolution & 512 pixels \\
\hline $\begin{array}{l}\text { Machine (optimum 64-slice } \\
\text { scanner) }\end{array}$ & CT GE Medical Systems Bright Speed S \\
\hline Hounsfield units & -1024 to 3071 \\
\hline Slice distance & $0.6250 \mathrm{~mm}$ \\
\hline Slice thickness & $0.6250 \mathrm{~mm}$ \\
\hline Spaces btwn slices & $2.500-3.750 \mathrm{~mm}$ \\
\hline kV & $100-120$ \\
\hline Exposure time & $1297-1397 \mathrm{msec}$ \\
\hline X-ray tube current & $129-149 \mathrm{~mA}$ \\
\hline Generator power & $13,000-18,000 \mathrm{VA}$ \\
\hline
\end{tabular}

now advanced to the new target position by the advanced software tools. To obtain the best possible correction, the bony elements were moved in relation to each other in various constellations and always also in relation to the reference data (Fig. 4A and B). The best matching arrangement was then selected to run a prediction algorithm to simulate soft-tissue changes. This simulation is not completely accurate but provides a useful estimation for the intensity and direction of expected transformations across the forehead and orbit (Fig. 4C). The final planning version (Fig. 5 ) was then selected as the basis for the design of the $3 \mathrm{D}$ template of the frontal bone segments (Fig. 6A) and the orbital bandeau (Fig. 6B). The templates allow the accurate transfer of the virtual correction plan to the actual intraoperative correction procedure (Fig. 7). After using a positive template (where bone fragments are placed on the outside convex side) we determined that negative templates (Fig. 7) were more applicable intraoperatively. These templates allowed for a precise reconstruction and plating of the orbital bandeau (Fig. 7).

\section{D Printing Process}

All casts and cutting guides were fabricated from medical-grade material (polyamide, PA-2200; EOS) and under certified production procedures (ISO [International Organization for Standardization] 9001 and ISO 13485) via selective/direct laser sintering/laser melting (Composites Busch S.A.). The advantages of this fabrication method are fast availability and secure sterilization without the risk of deformation or distortion. A test with less-expensive acrylonitrile butadiene styrene material proved to offer less intraoperative flexibility because of the need to cover the templates with sterile bags. Because of the chemical and thermal resistance of the polyamide 3D printouts, a steam sterilization process can be performed in an autoclave without any problems.

\section{Intraoperative Technique}

After the preoperative virtual planning process is completed, the plan is demonstrated and discussed with the family, with the aid of the CAD/CAM workstation for visualization. Thereafter, the manufacturing of the surgical templates is completed, and the patient is taken to the operating room, prepared, and draped as usual. The surgical procedure is performed by a team of pediatric neurosurgeons and craniomaxillofacial surgeons. Access to the cranial vault is done through a zigzag-patterned coronal incision, preserving the pericranium by dissection of the galeal flap. The 3D printed and preoperatively sterilized frontal and orbital cutting guides are placed on the cranial bone. Afterward, the planned osteotomy lines are marked using a surgical marking pen (gentian violet ink) (Fig. 6A and B). A bifrontal craniotomy is performed in standard fashion, while the osteotomies of the orbital bandeau are performed using a piezoelectric device (Piezosurgery system, Mectron Medical Technology). The temporal muscles are bilaterally minimally mobilized to enable the preparation of the lateral struts of the orbital bandeau guided by the suitable cutting templates (Fig. 6B). Additional osteotomies are performed on a separate table ac- 


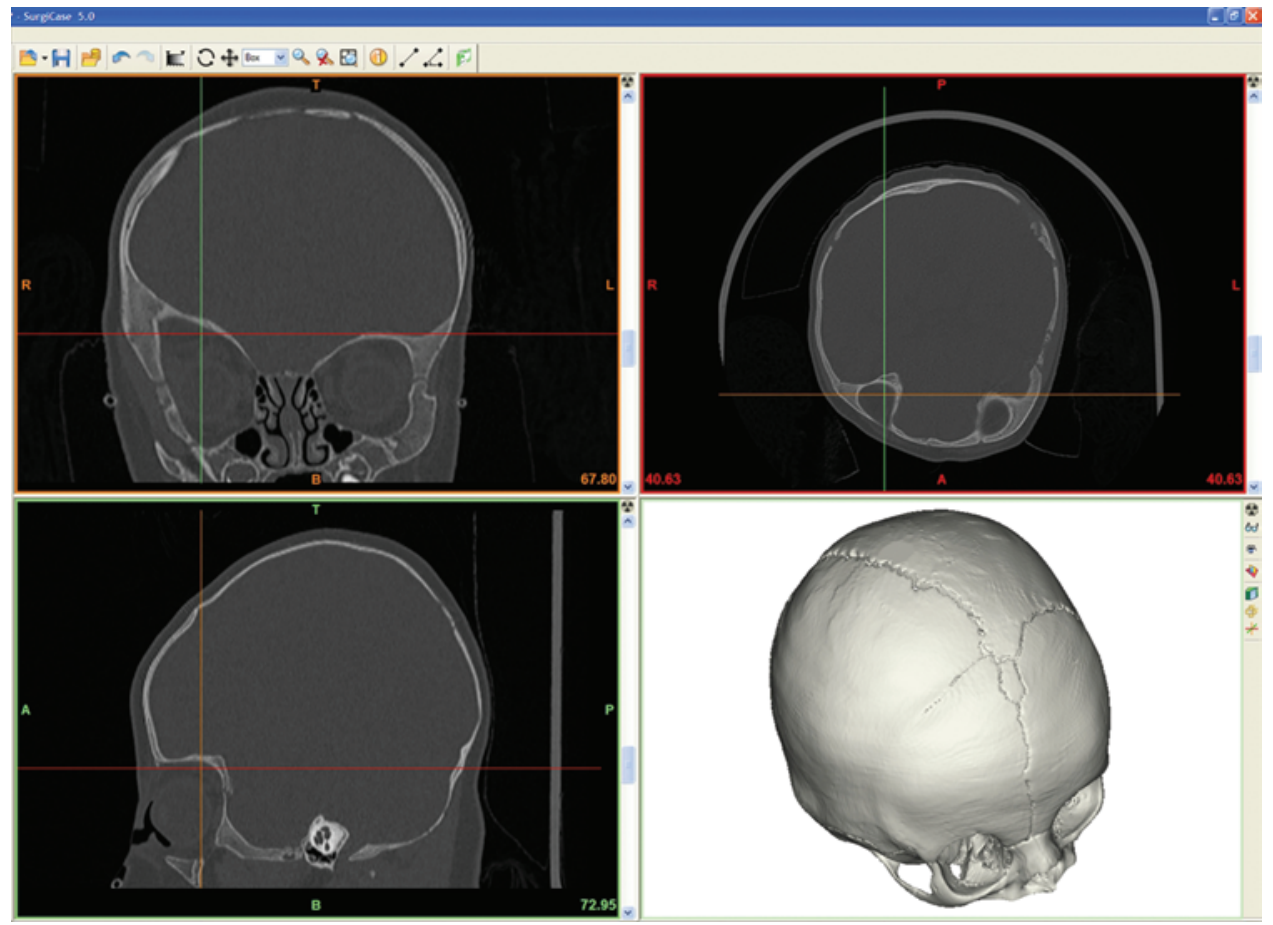

FIG. 1. CT images and the 3D reconstruction of these images using the SurgiCase-CMF software.

cording to the preoperatively planned virtual osteotomies (Fig. 7A). Afterward, the negative templates are used as guides for the fixation of the bone segments according to the preoperatively planned position (Fig. 7). These guides are designed as negative templates with edges for easy orientation and precise rigid bone fixation by absorbable poly-DL-lactic acid (PDLLA) fixation plates and screws

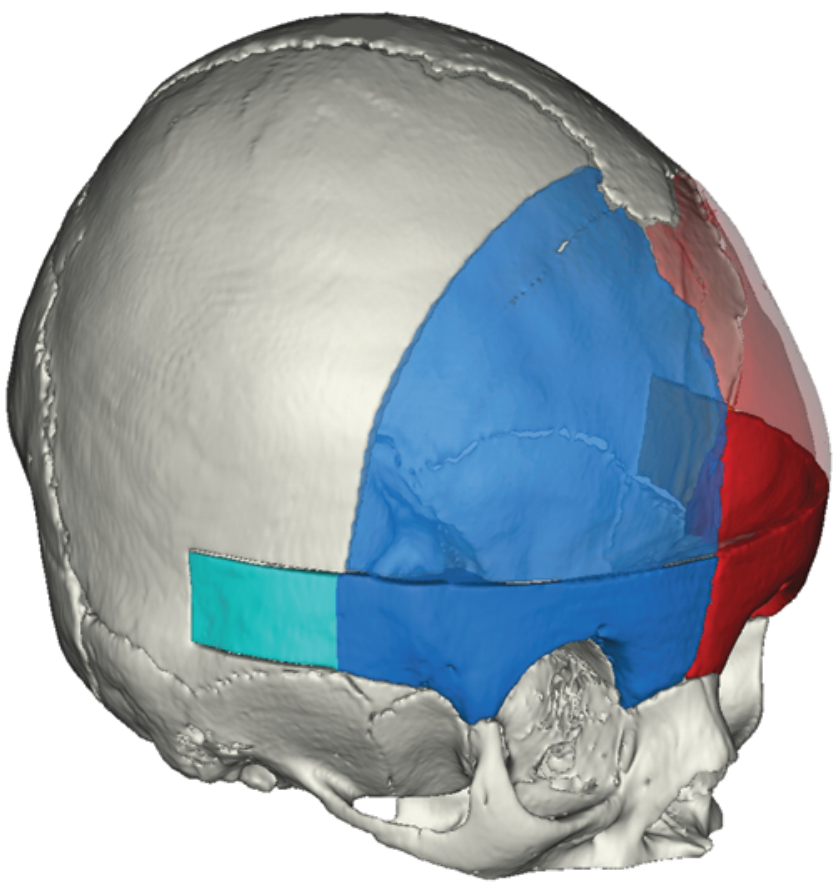

FIG. 2. Determination of the frontal craniotomy and frontoorbital advancement osteotomy.
(SonicWeld, KLS Martin) (Figs. 7B-D and 8A). First, the remodeled orbital bandeau is positioned and fixed in the new symmetrical position. The degree of overcorrection on the side ipsilateral to the craniosynostosis still depends on the surgeon's experience. However, the virtual plan will allow us to quantify the degree of overcorrection. Second, the frontal bone plates are placed and fixed by PDS sutures (Ethicon) (Fig. 8B). At the end of the surgical procedure, subgaleal drains without suction are inserted. Skin closure is done using multilayered resorbable closure.

\section{Results}

Based on our preliminary intraoperative experience, the virtually planned and 3D printed templates provide excellent accuracy for cranial modeling. They allow advanced cutting geometries, and every cut line for the frontal and frontoorbital osteotomies can be precisely defined by placing the templates on the skull (Fig. 6). After completing the frontal craniotomy and the osteotomy of the frontoorbital region, the bone fragments can be placed into the 3D printed positioning/shaping templates, which are easy and safe to handle (Fig. 7). In the printed negative form, the bone pieces can be fixed with resorbable plates and screws (Fig. 7), allowing a precise reconstruction based on the virtual planning data. We found that the bone fragments fit very well into the negative form. In our first experience, the 3D templates simplified and sped up the reconstruction process.

\section{Planning Time and Cost}

The planning went through many iterations for process improvement. The planning time took up to 6 working days, requiring many manual steps. After a suitable 

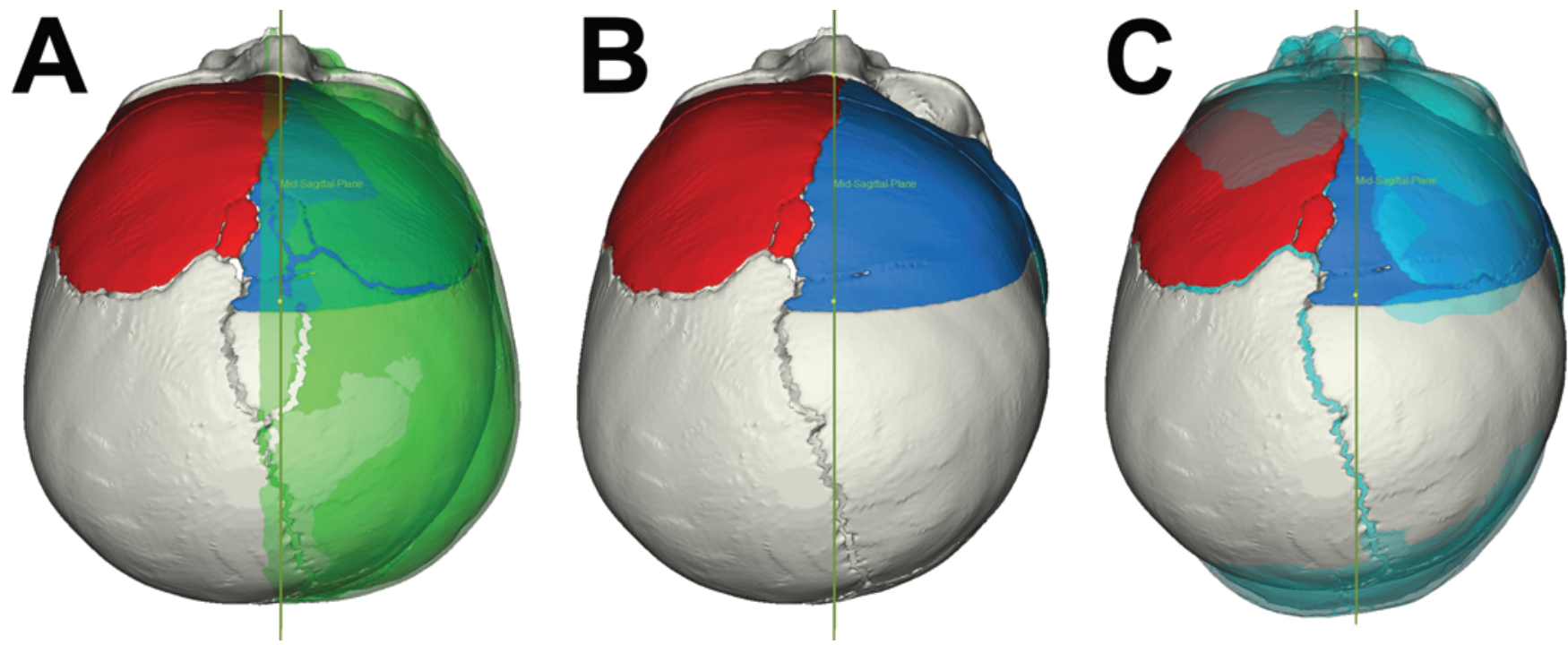

FIG. 3. A and B: Planning the surgical correction by mirroring the patient's unaffected side. C: Similar reference skull of the same age, sex, and ethnicity that is also used for planning the surgical correction.

workflow for all virtual procedures (such as the handling, import, and 3D reconstruction of image data), the planning procedure, and the design of placing and positioning/ shaping templates is well defined, the required planning time per patient is expected to be 1-2 working days.

Developing the planning tool and performing the virtual planning was performed by one author (J.B.). The cost of planning will largely depend on the complexity of the case but is expected to be the salary for 1-2 working days and overhead cost for the 3D printing. These costs can range between $\$ 3000$ and $\$ 4500$ (US dollars). The cost for materials is between $\$ 1500$ and $\$ 2000$.

\section{Case Descriptions}

Case 1

This 8-month-old girl had a nonsyndromal right-sided coronal craniosynostosis leading to plagiocephaly and brachycephaly. The patient presented with a typical Harlequin deformity of her right eye, flattening of her right forehead, closed fontanels, and a deviation of her nose root. Her head circumference was $43 \mathrm{~cm}$ (75th percentile). No deficits were seen during neurological examination, except a strabismus of the right eye, which had been present since birth. The patient's psychomotor development was normal. Bilateral frontoorbital advancement was conducted as described above. Intraoperative blood loss was 300 $\mathrm{ml}$, and $80 \mathrm{ml}$ of blood was transfused intraoperatively. The operative time was 300 minutes. The postoperative period was uneventful, and the patient was discharged on the 4th postoperative day. The postoperative follow-up after 3 weeks showed no neurological deficits, good scar healing, and very good reconstructive results on the right side, in spite of minimal swelling above of the left orbit.
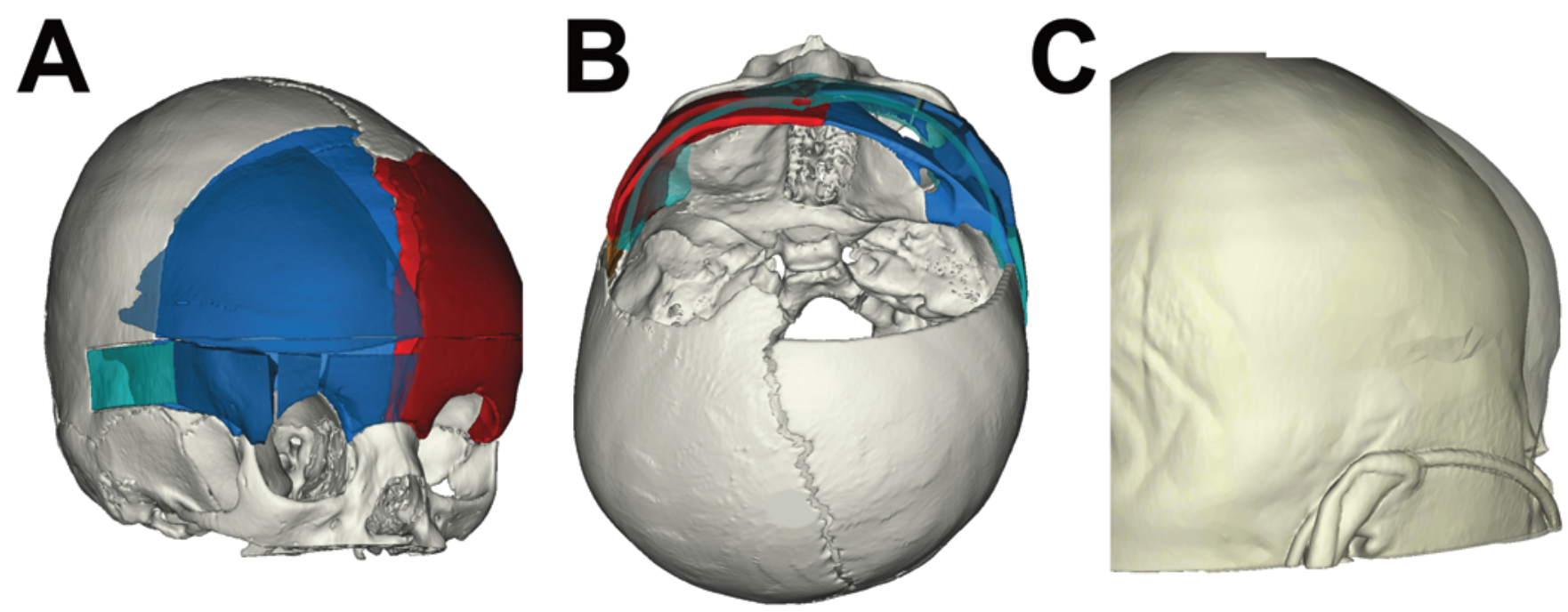

FIG. 4. A and B: After moving the single elements in relation to each other in various constellations, the best matching arrangement is selected. C: Image showing the proper estimation for the intensity and direction of expected transformations across the hemispheres. 


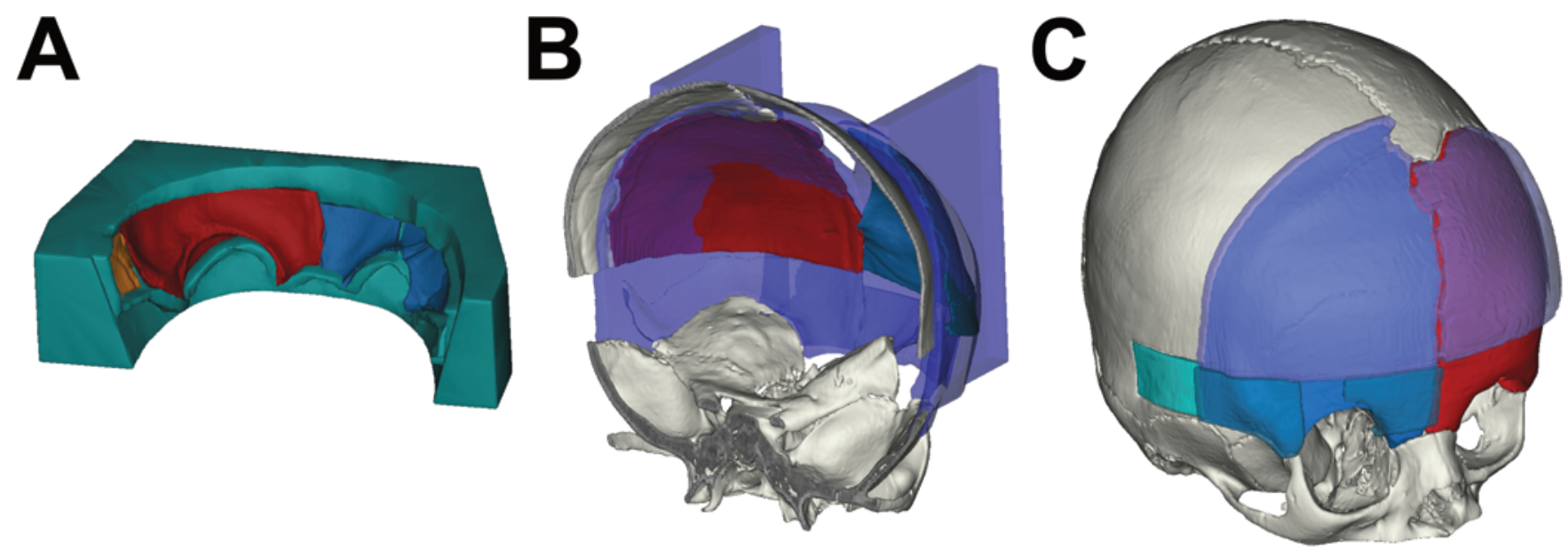

FIG. 5. A and B: Design of intraoperative applicable and 3D printable templates for the orbital bandeau and the frontal bone segments. C: Surgical cutting guides for alignment during the phase of surgical osteotomy of the single segments.

Additional follow-up with a 3D photo scan is planned 6 months postoperatively.

\section{Case 2}

This 8-month-old girl had a nonsyndromal right-sided coronal craniosynostosis leading to plagiocephaly. The patient presented with a typical Harlequin deformity of her right eye, flattening of her right forehead, closed fontanels, and a deviation of her nose root (Fig. 9A). Her head circumference was $40 \mathrm{~cm}$ (50th percentile). No deficits were seen during neurological examination, except a strabismus of the right eye, which had been present since birth. The patient's psychomotor development was normal. Bilateral frontoorbital advancement was conducted as described above. Intraoperative blood loss was $500 \mathrm{ml}$, and $150 \mathrm{ml}$ of blood was transfused intraoperatively. The operative time was 270 minutes. The postoperative period was uneventful, and the patient was discharged on the 4th postoperative day. The postoperative follow-up after 3 weeks showed no neurological deficits, good scar healing, and very good reconstructive results on the right side (Fig. 9B). A light asymmetry with residual right-sided flattening of the forehead is still visible; however, given the short follow-up time, it is expected to improve with time. An additional follow-up with a 3D photo scan is planned 6 months postoperatively.

\section{Discussion}

Currently, template- and CAD/CAM-based bone remodeling for craniosynostosis surgery is an emerging topic, with a few novel techniques described in the literature over the last few years. ${ }^{1,2,8-10,12,16,18,19,22,25,26}$ Still, surgical procedures for craniosynostosis lack methods simplifying and objectifying the cranial remodeling process. The intraoperative process and its cosmetic outcome continue to be determined mostly by the surgeon's experience and learning curve. Marchac, one of the first to acknowledge this problem, developed a frontal pattern in 1983 to aid in remodeling the frontal bandeau, known as Marchac forehead templates. ${ }^{13}$ Though proven to be very helpful at the time, these templates cannot account for individual variations. Mommaerts et al. advocated a simple forehead template, mainly used as an osteotomy guideline, since the shape of the template is approximate and not based on normative data. ${ }^{18}$ Pappa et al. created a frontal bandeau template that was adjusted preoperatively on the child's forehead, sterilized, and used intraoperatively, yet the
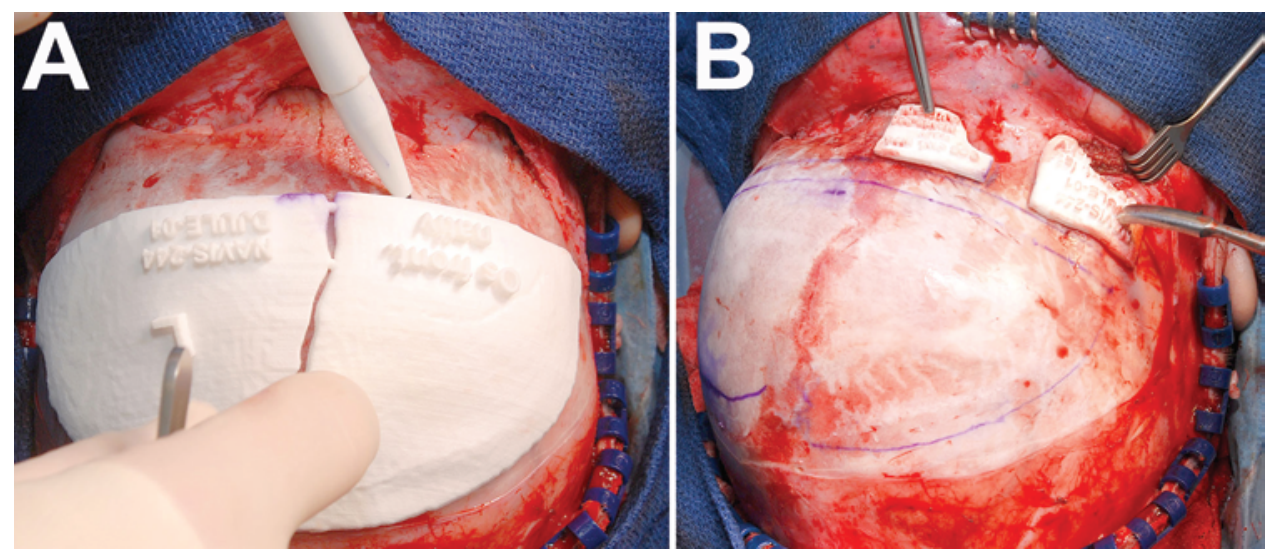

FIG. 6. A: Marking of planned osteotomies on the skull guided by the preoperatively manufactured cutting template. B: Various cutting 3D printed guides for the segmental osteotomy. 

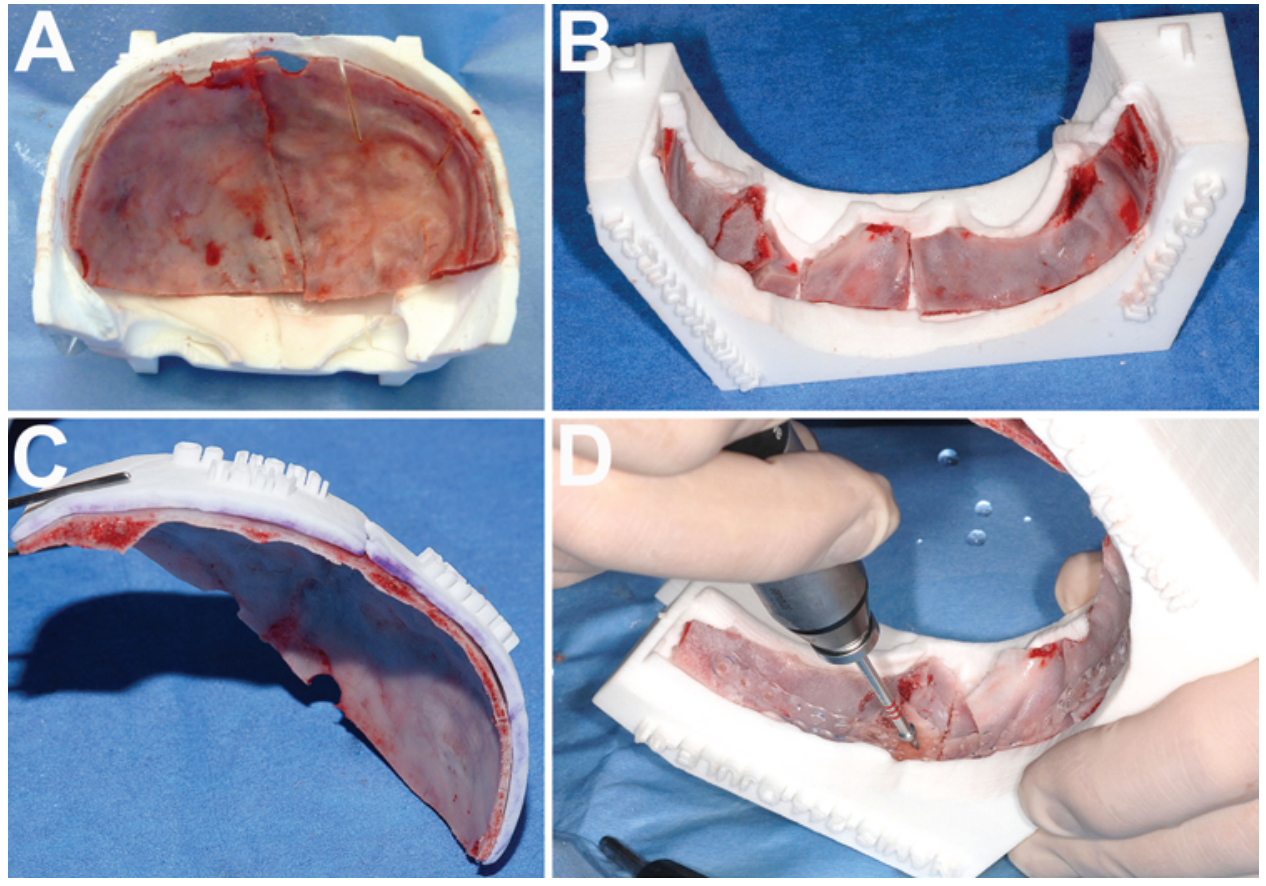

FIG. 7. A: Ex vivo shaping/positioning guide for bone segments. The guide is bowl-shaped with ridges marking the correct position of different bone segments in their new position. B: Final positioning template for orbital bandeau fixation by internally placed resorbable plates and screws. C: The precise fabrication of the 3D printed templates. D: Fixation of the ex vivo shaped bone segments in the final position by resorbable PDLLA plates while still resting in the positioning guide.

adjustment and the resulting shape were based on subjective judgment only. ${ }^{19}$ The template used by Burstein et al., which employed a LactoSorb strip as a Marchac template by modulating it in a heated in water bath, was obviously also influenced by the surgeon's subjective intraoperative assessment. ${ }^{2}$ Saber et al. designed a frontal bandeau template out of steel based on 103 normal infant skulls, which was then adjusted for size and age via 3D computeraided design and machining methods. ${ }^{10,22}$ The individual template was then used intraoperatively, leading to more objective reconstruction of the frontal bandeau. Although the templates were individualized for each patient and used intraoperatively, the template provides objective reconstruction only for the frontal bandeau without addressing the reconstruction of the frontal bone. Similarly, Shah et al. described an individualized set of templates for the reconstruction of the frontal bandeau. ${ }^{26}$ The first template fits along the trigonocephalic supraorbital region to aid in the osteotomy placement, the second acts as an ex vivo guide to fashion and stabilize the segments, and the third is placed posterior to the temporal extension of the frontoorbital segment and helps establish the magnitude of the needed advancement. ${ }^{26}$ Imai et al. described a distraction device to gradually treat craniosynostosis. ${ }^{9}$ They used a solid 3D model to determine preoperatively the most favorable osteotomy lines and to determine the vectors and amount of advancement for distraction. However, the remodeling is based on preoperative simulation that does not include intraoperative frame-based remodeling, and a second operation is needed to remove the device. Hochfeld et al. developed an intraoperative reconstruction tool for cranial remodeling based on an evolving database of normal head shapes. ${ }^{8}$ This tool is capable of transmitting statistical information into the actual surgi-

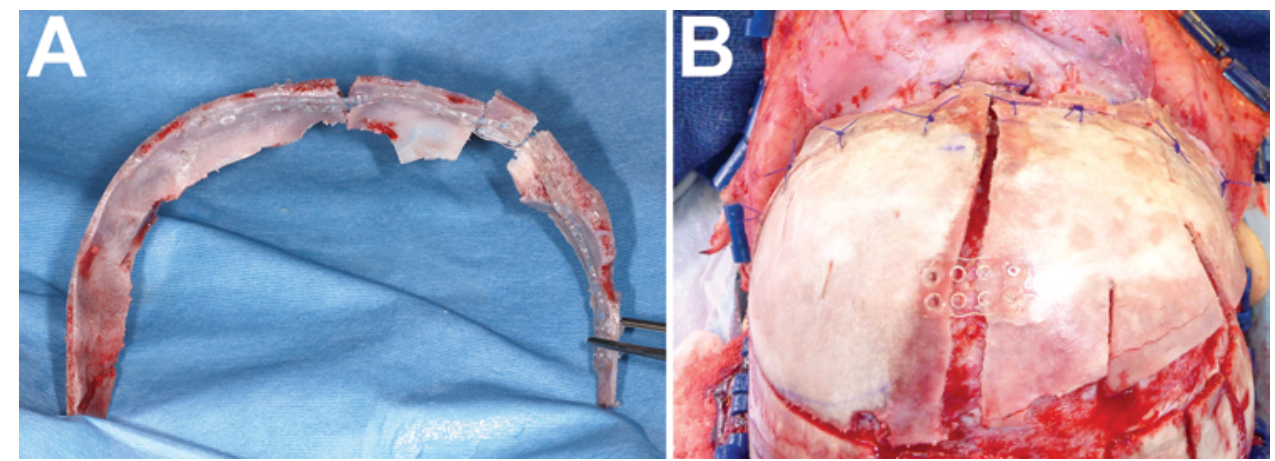

FIG. 8. A: Final appearance of the orbital bandeau after fixation with resorbable PDLLA plates from the interior side of the bone. B: Fixation of the ex vivo shaped and fixated bone segments in the final position by resorbable PDLLA plates, screws, and PDS sutures. 


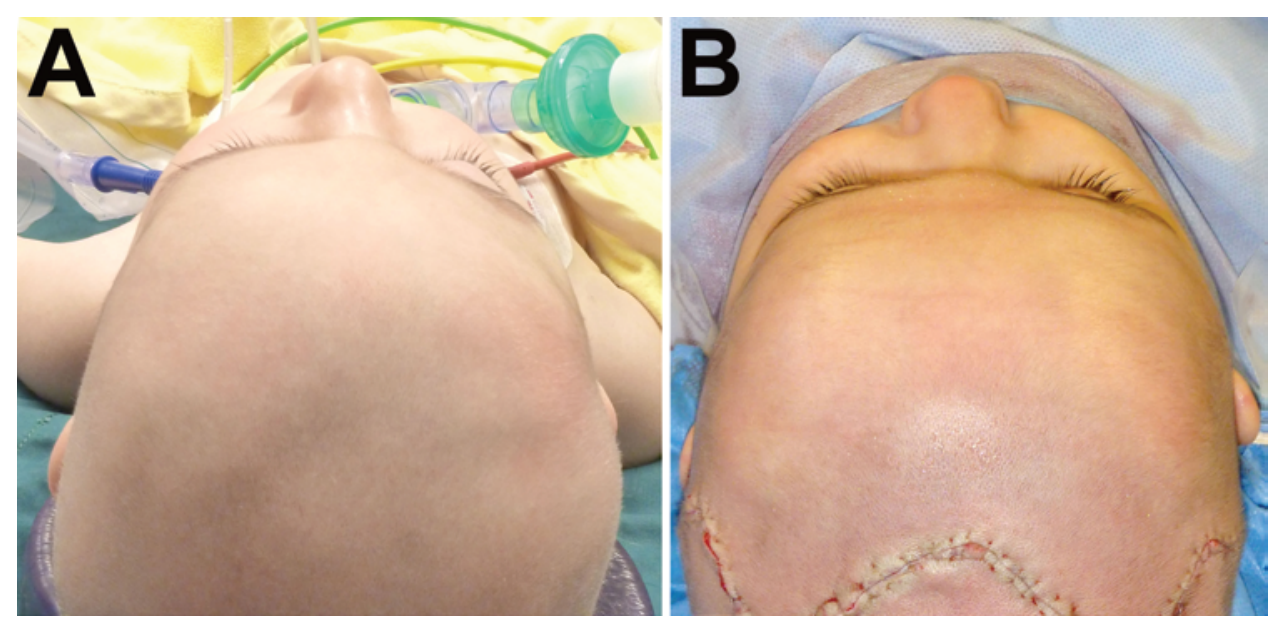

FIG. 9. Case 2. Photographs obtained of an 8-month-old girl with a nonsyndromal right-sided coronal craniosynostosis leading to a plagiocephaly. A: Harlequin deformity of the right eye and flattening of the right forehead is seen. B: Postoperative result showing good correction of the Harlequin deformity, flattening of the forehead, and deviation of the nose root.

cal procedure, leading to more objective surgical results; it provides frame-based reconstruction for the frontal bone as well. However, its setup and usage are time consuming and complex and might lead to a longer operation time, which correlates with increased blood loss. ${ }^{6}$ Seruya et al. and Mardini et al. described cranial vault remodeling with prefabricated templates using the preoperative $\mathrm{CAD} / \mathrm{CAM}$ technique. ${ }^{16,25}$ Both groups concluded that this method allows for more precise, accurate, efficient, and rapid surgery, without the need for subjective assessment of the desired calvarial shape. In addition, it accelerates the learning curve for young and less experienced surgeons and helps families gain a better understanding of the disease and the surgery. ${ }^{16,25}$ These observations have been made by us as well.

The surgical molding of the bone is only one of the variables affecting the long-term cosmetic outcome in craniosynostosis. The degree of the cranial base deformity, which is not corrected through the frontoorbital advancement, might have an impact on long-term outcome. ${ }^{3}$ The timing of surgery has also been shown to be an important variable that influences the relapse rate., ${ }^{6,27}$ According to Foster et al. and Marchac and Renier, patients who undergo surgery between the ages of 6 and 12 months have the best outcome and lowest recrudescence rate. ${ }^{5,14}$ For this reason, children with coronal craniosynostosis undergo surgery at our institution at the age of 6-12 months. Other factors affecting outcome and relapse rate might be the devascularization of the frontoorbital bandeau and the patient's genetic makeup. These variables might be more influential on outcome than the surgical correction of craniosynostosis.

Overcorrection of the side ipsilateral to the craniosynostosis has been advocated by some surgeons to account for recrudescence of the synostosis. ${ }^{4,17}$ The degree of overcorrection remains a variable that is difficult to quantify and largely depends on the surgeon's experience. After adjusting and plating the orbital bandeau within the negative mold (Fig. 7), the assembled bandeau is put in place and the overcorrection is performed. We measure the overcorrection intraoperatively and correlate it to the clinical out- come and the 3D photographs with the goal of reaching a better standardization of the advancement. These data are integrated in future treatment planning.

Our novel technique using 3D printed templates for the frontal bandeau and the frontal bone, based on computerized calculations for the ideal cosmetic results of each individual patient, seems to provide an overall easy and feasible intraoperative solution. From our initial experience in 2 cases, the 3D printed templates are very easy to handle intraoperatively, leave almost no room for subjective decision making concerning the cranial remodeling, and lead to very satisfying results with a steep learning curve and no prolongation of the operation time. The main difference of our method compared with the above-described procedures is the fabrication of negative molds, which allow shaping the orbital bandeau and fixing it with the resorbable plates. We believe that the combination of cutting and positioning/shaping templates allow a more precise reconstruction.

\section{Limitations}

Our experience so far is based on only 2 cases and lacks long-term follow-up of patients. This paper only describes the novel technical aspects and is illustrated by a representative case. At this time, no conclusions can be drawn on surgical safety, long-term results, and potential reduction in relapse rates. The preoperative planning is time consuming and requires knowledge of the software (SurgiCase-CMF, version 5.0, Materialise). The process depends on 3D printing capabilities, which also potentially limits the feasibility. The cost of virtual surgery planning and manufacturing of the 3D models is approximately $\$ 4500$ $\$ 6000$ (US dollars). The cost will also largely depend on the complexity of the case and the number of templates.

\section{Conclusions}

Our novel technique using 3D printed templates for frontoorbital advancement surgery will potentially allow for a more predictable reconstruction based on standardized measurements. Based on our first cases, this technique 
appears to be safe and feasible. To determine whether we are able to achieve shortened surgical times and improved long-term results, a larger series of patients is needed.

\section{References}

1. Burge J, Saber NR, Looi T, French B, Usmani Z, Anooshiravani N, et al: Application of CAD/CAM prefabricated agematched templates in cranio-orbital remodeling in craniosynostosis. J Craniofac Surg 22:1810-1813, 2011

2. Burstein F, Eppley B, Hudgins R, Williams J, Boydston W, Reisner A, et al: Application of the spanning plate concept to frontal orbital advancement: techniques and clinical experience in 60 patients. J Craniofac Surg 17:241-245, 2006

3. Calandrelli R, D’Apolito G, Gaudino S, Stefanetti M, Massimi L, Di Rocco C, et al: Radiological assessment of skull base changes in children with syndromic craniosynostosis: role of "minor" sutures. Neuroradiology 56:865-875, 2014

4. Engel M, Castrillon-Oberndorfer G, Hoffmann J, Mühling J, Seeberger R, Freudlsperger C: Long-term results in nonsyndromatic unilateral coronal synostosis treated with frontoorbital advancement. J Craniomaxillofac Surg 41:747-754, 2013

5. Foster KA, Frim DM, McKinnon M: Recurrence of synostosis following surgical repair of craniosynostosis. Plast Reconstr Surg 121:70e-76e, 2008

6. Guzman R, Looby JF, Schendel SA, Edwards MS: Frontoorbital advancement using an en bloc frontal bone craniectomy. Neurosurgery 68 (1 Suppl Operative):68-74, 2011

7. Hankinson TC, Fontana EJ, Anderson RC, Feldstein NA: Surgical treatment of single-suture craniosynostosis: an argument for quantitative methods to evaluate cosmetic outcomes. J Neurosurg Pediatr 6:193-197, 2010

8. Hochfeld M, Lamecker H, Thomale UW, Schulz M, Zachow S, Haberl H: Frame-based cranial reconstruction. J Neurosurg Pediatr 13:319-323, 2014

9. Imai K, Komune H, Toda C, Nomachi T, Enoki E, Sakamoto $\mathrm{H}$, et al: Cranial remodeling to treat craniosynostosis by gradual distraction using a new device. J Neurosurg 96:654-659, 2002

10. Khechoyan DY, Saber NR, Burge J, Fattah A, Drake J, Forrest CR, et al: Surgical outcomes in craniosynostosis reconstruction: the use of prefabricated templates in cranial vault remodelling. J Plast Reconstr Aesthet Surg 67:9-16, 2014

11. Lwin CT, Richardson D, Duncan C, May P: Relapse in fronto-orbital advancement: a pilot study. J Craniofac Surg 22:214-216, 2011

12. Marchac D: A frontal pattern for frontocranial remodeling. Ann Plast Surg 17:263-265, 1986

13. Marchac D: Radical forehead remodeling for craniostenosis. Plast Reconstr Surg 61:823-835, 1978

14. Marchac D, Renier D: Craniosynostosis. World J Surg 13: 358-365, 1989

15. Marcus JR, Domeshek LF, Loyd AM, Schoenleber JM, Das RR, Nightingale RW, et al: Use of a three-dimensional, normative database of pediatric craniofacial morphology for modern anthropometric analysis. Plast Reconstr Surg 124:2076-2084, 2009

16. Mardini S, Alsubaie S, Cayci C, Chim H, Wetjen N: Threedimensional preoperative virtual planning and template use for surgical correction of craniosynostosis. J Plast Reconstr Aesthet Surg 67:336-343, 2014

17. Mesa JM, Fang F, Muraszko KM, Buchman SR: Reconstruction of unicoronal plagiocephaly with a hypercorrection surgical technique. Neurosurg Focus 31(2):E4, 2011

18. Mommaerts MY, Janssens G, Jans G, Vander Sloten J, Van der Perre G: Clinical validation of individual templates in cranial reconstruction. Int Congr Ser 1256:1263-1268, 2003

19. Pappa H, Richardson D, Webb AA, May P: Individualized template-guided remodeling of the fronto-orbital bandeau in craniosynostosis corrective surgery. J Craniofac Surg 20: 178-179, 2009

20. Renier D, Sainte-Rose C, Marchac D, Hirsch JF: Intracranial pressure in craniostenosis. J Neurosurg 57:370-377, 1982

21. Rodt T, Schlesinger A, Schramm A, Diensthuber M, Rittierodt M, Krauss JK: 3D visualization and simulation of frontoorbital advancement in metopic synostosis. Childs Nerv Syst 23:1313-1317, 2007

22. Saber NR, Phillips J, Looi T, Usmani Z, Burge J, Drake J, et al: Generation of normative pediatric skull models for use in cranial vault remodeling procedures. Childs Nerv Syst 28:405-410, 2012

23. Salyer KE, Hall JD: Bandeau - the focal point of frontocranial remodeling. J Craniofac Surg 1:18-31, 1990

24. Selber JC, Brooks C, Kurichi JE, Temmen T, Sonnad SS, Whitaker LA: Long-term results following fronto-orbital reconstruction in nonsyndromic unicoronal synostosis. Plast Reconstr Surg 121:251e-260e, 2008

25. Seruya M, Borsuk DE, Khalifian S, Carson BS, Dalesio NM, Dorafshar AH: Computer-aided design and manufacturing in craniosynostosis surgery. J Craniofac Surg 24:1100-1105, 2013

26. Shah A, Patel A, Steinbacher DM: Simulated frontoorbital advancement and intraoperative templates enhance reproducibility in craniosynostosis. Plast Reconstr Surg 129:1011e$1012 \mathrm{e}, 2012$

27. Utria AF, Mundinger GS, Bellamy JL, Zhou J, Ghasemzadeh A, Yang R, et al: The importance of timing in optimizing cranial vault remodeling in syndromic craniosynostosis. Plast Reconstr Surg [epub ahead of print], 2014

28. Whitaker LA, Schut L, Kerr LP: Early surgery for isolated craniofacial dysostosis. Improvement and possible prevention of increasing deformity. Plast Reconstr Surg 60:575-581, 1977

\section{Author Contributions}

Conception and design: Guzman, Soleman, Thieringer. Acquisition of data: Soleman. Analysis and interpretation of data: Soleman, Thieringer, Beinemann. Drafting the article: Soleman, Thieringer, Beinemann. Critically revising the article: Guzman, Kunz. Reviewed submitted version of manuscript: all authors. Approved the final version of the manuscript on behalf of all authors: Guzman. Statistical analysis: Soleman, Thieringer. Study supervision: Guzman, Kunz.

\section{Correspondence}

Raphael Guzman, Department of Neurosurgery, University Hospital of Basel, Spitalstrasse 21, 4031 Basel, Switzerland. email: raphael.guzman@usb.ch. 